\title{
Prognostic significance of the mRNA expression of ERCC1, RRM1, TUBB3 and TYMS genes in patients with non-small cell lung cancer
}

\author{
SHENGJIE SUN*, WEIWEI SHI*, ZHIYONG WU, GUOQING ZHANG, BO YANG and SHUNCHANG JIAO \\ Department of Oncology, The General Hospital of People's Liberation Army, Beijing 100853, P.R. China
}

Received December 11, 2013; Accepted April 29, 2015

DOI: $10.3892 / \mathrm{etm} .2015 .2636$

\begin{abstract}
The present study aimed to investigate the prognostic value of excision repair cross-complementing 1 (ERCC1), ribonucleotide reductase subunit M1 (RRM1), class III $\beta$-tubulin (TUBB3) and thymidylate synthase (TYMS) in patients with non-small cell lung cancer (NSCLC) receiving platinum-based adjuvant chemotherapy. The mRNA expression of these genes was assessed in 72 tumor tissue samples obtained following surgery, using multiplex branched-DNA technology. Subsequent to surgery, all 72 patients with NSCLC were treated with platinum-based chemotherapy. The expression of these five genes was analyzed and the correlation with clinical characteristics and patient survival was investigated. Among the 72 samples, the incidence rate of mRNA expression of ERCC1 was $38.9 \%$ (28/72), RRM1 was $55.6 \%$ (40/72), TUBB3 was $47.2 \%(34 / 72)$ and TYMS was $62.5 \%$ (45/72). The incidence rate of ERCC1 expression in adenocarcinoma (34.2\%) was significantly lower than that in non-adenocarcinoma (44.1\%; $\mathrm{P}<0.05)$. Furthermore, the incidence rates of $T Y M S$ and $T U B B 3$ expression in the high-median differentiation tissue samples were significantly lower than those in the low differentiation tissue samples $(\mathrm{P}<0.05)$. When the correlation of gene expression and patient survival was analyzed, high expression of ERCC1, RRM1, TUBB3 or TYMS was found to be associated with poor prognosis $(\mathrm{P}<0.001, \mathrm{P}=0.001, \mathrm{P}=0.001$ and $\mathrm{P}=0.001$, respectively). ERCC1, RRM1, TUBB3 and TYMS are key factors involved in survival following surgical treatment in patients with NSCLC. The mRNA expression of these genes may have prognostic value for patients with NSCLC treated with platinum-based chemotherapy.
\end{abstract}

Correspondence to: Professor Shunchang Jiao, Department of Oncology, The General Hospital of People's Liberation Army, 28 Fuxing Road, Beijing 100853, P.R. China

E-mail: wenzhangbj001@sina.cn

*Contributed equally

Key words: excision repair cross-complementing 1, ribonucleotide reductase subunit M1, class III $\beta$-tubulin, thymidylate synthase, non-small cell lung cancer

\section{Introduction}

Lung cancer is one of the most prevalent types of malignant tumor in China and the overall five-year survival rate remains unsatisfactory. Approximately $80-85 \%$ of all lung cancer diagnoses are non-small cell lung cancer (NSCLC) (1). Adjuvant chemotherapy, for example platinum agents combined with cytotoxic agents, including vinorelbine, gemcitabine and taxane, has become a treatment standard for NSCLC (2). However, the resistance of tumor cells to these drugs leads to a poor prognosis and survival rate. Therefore, the investigation of tumor biomarkers associated with resistance to chemotherapeutic drugs is required.

Excision repair cross-complementing 1 (ERCC1) is a structure-specific DNA repair endonuclease responsible for the 5'-incision during DNA excision repair. Clinical studies have indicated that high ERCCl expression is associated with resistance to platinum-based chemotherapy (3). The ribonucleotide reductase subunit M1 (RRMI) gene, located on chromosome 11p15.5, encodes one of two non-identical subunits of the ribonucleoside-diphosphate reductase. The ribonucleoside-diphosphate reductase enzyme is essential for the production of deoxyribonucleotides prior to DNA synthesis. It has been reported that NSCLC patients with low $R R M 1$ expression have improved survival when treated with gemicitabine-based therapy (4). Thymidylate synthase (TYMS) is the enzyme used to generate thymidine monophosphate (dTMP), which is subsequently phosphorylated to thymidine triphosphate for use in DNA synthesis and repair. TYMS inhibitors, including fluorinated pyrimidine derivatives, are capable of inhibiting the activity of TYMS. Thus, TYMS expression is associated with in vivo chemosensitivity to TYMS inhibitors (5). Class III $\beta$-tubulin (TUBB3) encodes a class III member of the $\beta$-tubulin protein family. The overexpression of $T U B B 3$ is associated with resistance to docetaxel and paclitaxel $(6,7)$.

Changes in the mRNA expression of the above tumor biomarkers during tumor development may have an impact on the prognosis of patients with NSCLC. In the present study, multiplex branched-DNA liquidchip technology was used to analyze the mRNA expression of the ERCCl, RRMI, TUBB3 and TYMS genes in tumor tissue samples from patients with resected NSCLC prior to chemotherapy. The multiplex branched-DNA technology is a sandwich nucleic 
acid hybridization method in which targets are captured through cooperative hybridization of multiple probes, then coupled with a fluorescence signal amplification system $(8,9)$. The signals were detected using the Luminex ${ }^{\circledR} 200$ system (Luminex, Austin, TX, USA). It is a multiplex assay that enables the measurement of the mRNA expression of $>30$ biomarkers in one test well (8). The present study focused on: i) the analysis of the correlation between gene expression and different clinical characteristics; and ii) the analysis of the correlation between gene expression and patient survival.

\section{Materials and methods}

Patient samples. A total of 72 patients with NSCLC at the General Hospital of People's Liberation Army (Beijing, China) who underwent curative surgery between March and October 2011 were enrolled in the present study. Informed consent was obtained from all patients. Patient information and clinical characteristics are shown in Table I. A total of 72 tissue samples were obtained following surgery and the tissues were fixed in $10 \%$ neutral formalin for $24 \mathrm{~h}$, desiccated and embedded in paraffin. We confirmed the hematoxylin and eosin staining results of the 72 formalin-fixed paraffin-embedded (FFPE) tissue samples from the pathology department, which revealed that the tumor sections contained large numbers of tumor cells (usually $>60 \%$ ). For each FFPE tissue sample, the tumor tissue was cut using microdissection techniques and sent to Guangzhou Surexam Medical Test Center (SurExam Bio-Tech Co., Ltd., Guangzhou, China) for ERCCl, RRM1, TUBB3 and TYMS mRNA expression analysis. This study was approved by the ethics comittee of the General Hospital of People's Liberation Army.

mRNA expression analysis of ERCC1, RRM1, TUBB3 and TYMS using multiplex branched-DNA liquidchip technology. Multiplex branched-DNA liquidchip technology measures mRNA expression without RNA extraction, reverse transcription or polymerase chain reaction amplification. FFPE tissue samples were homogenized at $65^{\circ} \mathrm{C}$ for $1 \mathrm{~h}$, then the supernatant was used for hybridization. Sandwich nucleic acid hybridization was performed for $16 \mathrm{~h}$ using magnetic capture beads containing probes for ERCC1, RRM1, TUBB3 and $T Y M S$, the homogenate and another set of capture probes for ERCC1, RRM1, TUBB3 and TYMS. The hybridization reaction was performed in a 96-well plate. The unbound RNA was removed by washing three times with $250 \mu \mathrm{l}$ wash buffer ( $0.1 \mathrm{X}$ saline sodium citrate and $0.03 \%$ lithium lauryl sulfate) under a vacuum system. Signal amplification steps were performed through incubation with $100 \mu \mathrm{l}$ pre-amplifier solution for $1 \mathrm{~h}$ at $50^{\circ} \mathrm{C}$, followed by incubation with $100 \mu 1$ amplifier solution for $1 \mathrm{~h}$ at $50^{\circ} \mathrm{C}$, then incubation with probes labeled with biotin for $1 \mathrm{~h}$ at $50^{\circ} \mathrm{C}$. Following washing, the samples were incubated with $100 \mu l$ streptavidin conjugated phycoerythrin solution at $50^{\circ} \mathrm{C}$ for $30 \mathrm{~min}$, prior to analysis using the Luminex 200 system (Luminex).

The fluorescence intensity value of ERCCl, RRM1, TUBB3 and TYMS generated using the Luminex 200 system was normalized to that of the house keeping genes, including $\beta$ 2-microglobulin, box binding protein and the transferrin receptor. The normalized values of ERCC1,RRM1,TUBB3 and
Table I. Patient information and clinical characteristics.

\begin{tabular}{lcc}
\hline Variable & No. of patients & Percentage \\
\hline Age & & \\
$<60$ years & 42 & 58.3 \\
$\geq 60$ years & 30 & 41.7 \\
Gender & & \\
Male & 47 & 65.3 \\
Female & 25 & 34.7 \\
Smoking & & \\
Yes & 42 & 58.3 \\
No & 30 & 41.7 \\
Histology & & \\
Adenocarcinoma & 38 & 52.8 \\
Non-adenocarcinoma & 34 & 47.2 \\
Differentiation & & \\
High-Mid & 40 & 58.3 \\
Low & 32 & 41.7 \\
Staging & & \\
I/II & 47 & 65.3 \\
III & 20 & 27.8 \\
IV & 5 & 6.9 \\
Node metastasis & & 34.7 \\
Yes & 25 & 65.3 \\
No & 47 & \\
\hline
\end{tabular}

TYMS were compared to the cut-off value of each gene, which was provided by Guangzhou Surexam Medical Test Center. ERCC1, RRM1, TUBB3 and TYMS mRNA expression was considered to be positive (high expression) if the normalized values were equal or higher than the cut-off value; otherwise, the result was considered to be negative (low expression).

Statistical analysis. Data were analyzed using the SPSS version 19.0 software package (SPSS, Inc., Chicago, IL, USA). The association between gene expression and different clinical characteristics was analyzed using the $\chi^{2}$ test. The correlation between mRNA expression levels was analyzed using Spearman correlation coefficients. The Kaplan-Meier method was used to analyze the correlation between gene expression and patient survival. $\mathrm{P}<0.05$ was considered to indicate a statistically significant difference.

\section{Results}

Correlation between ERCC1, RRM1, TUBB3 or TYMS mRNA expression and clinical characteristics. Tumor specimens from 72 patients with NSCLC were subjected to mRNA expression analysis. Among the 72 samples, the incidence rate of a high mRNA expression level of the ERCCl gene was $38.9 \%$ (28/72), RRM1 was $55.6 \%$ (40/72), TUBB3 was $47.2 \%$ (34/72) and TYMS was $62.5 \%$ (45/72; Table II). The correlation between the expression of these four genes and different clinical characteristics was assessed. The incidence rate of 


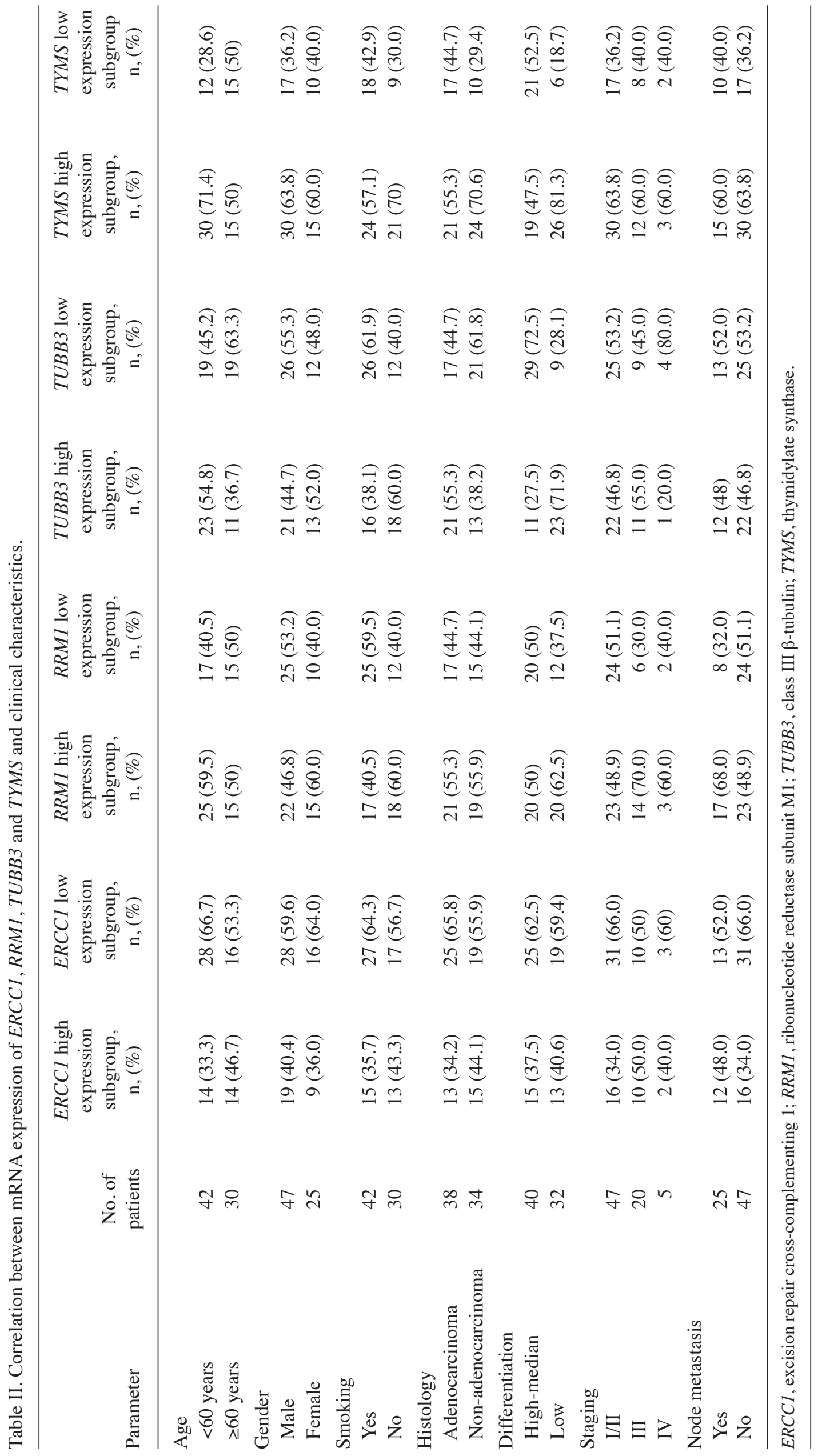




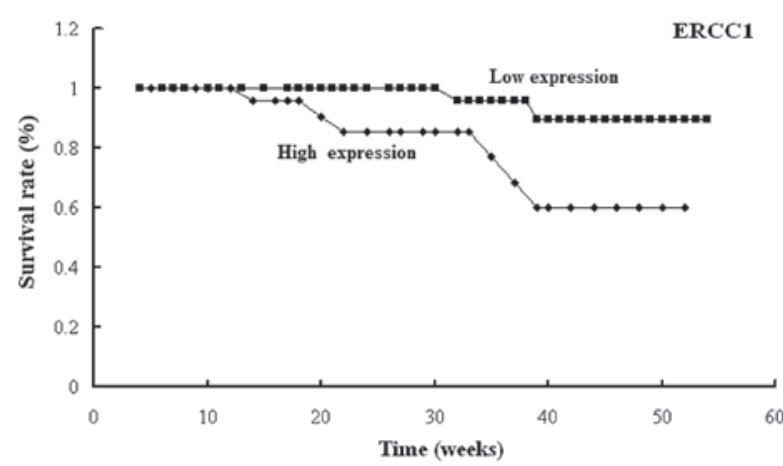

Figure 1. Survival curve following surgery in 72 patients with non-small cell lung cancer based on the mRNA expression of the ERCC1 gene. The one-year survival rates of the patients with high $E R C C 1$ expression and low $E R C C 1$ expression were 78.6 and $95.4 \%$, respectively. $\mathrm{P}<0.001$. ERCC1, excision repair cross-complementing 1.

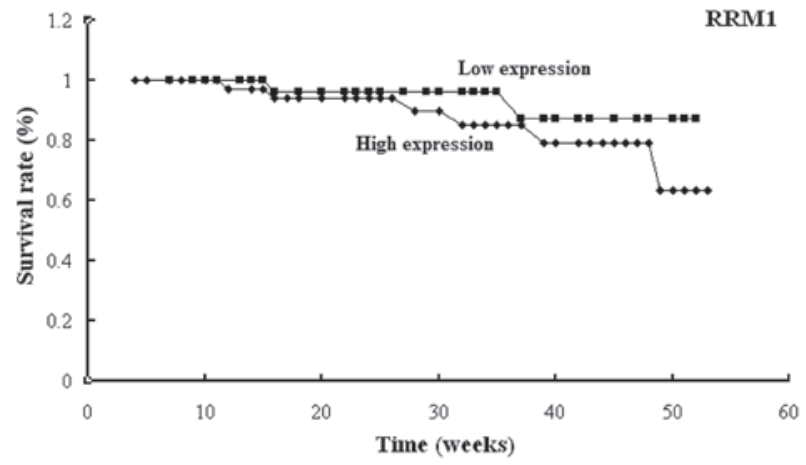

Figure 2. Survival curve following surgery in 72 patients with non-small cell lung cancer based on the mRNA expression of the $R R M 1$ gene. The one-year survival rates of the patients with high $R R M I$ expression and low $R R M I$ expression were 85.0 and $93.7 \%$, respectively. $\mathrm{P}=0.001$. $R R M 1$, ribonucleotide reductase subunit M1.

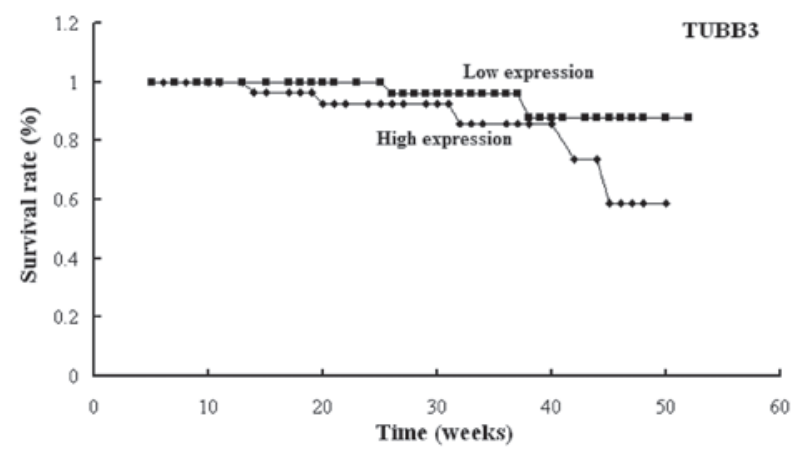

Figure 3. Survival curve following surgery in 72 patients with non-small cell lung cancer based on the mRNA expression of the TUBB3 gene. The one-year survival rates of the patients with high $T U B B 3$ expression and low TUBB3 expression were 85.3 and $94.7 \%$, respectively. $\mathrm{P}=0.001$. TUBB3, class III $\beta$-tubulin.

expression of the ERCC1 gene in adenocarcinoma (34.2\%) was found to be significantly lower than that in non-adenocarcinoma (44.1\%; $\mathrm{P}<0.05)$. Furthermore, the incidence rates of TYMS and TUBB3 expression in high-median differentiation tissue samples were observed to be significantly lower than those in low differentiation tissue samples $(\mathrm{P}<0.05)$. However, no correlation was observed between ERCC1, RRM1, TUBB3

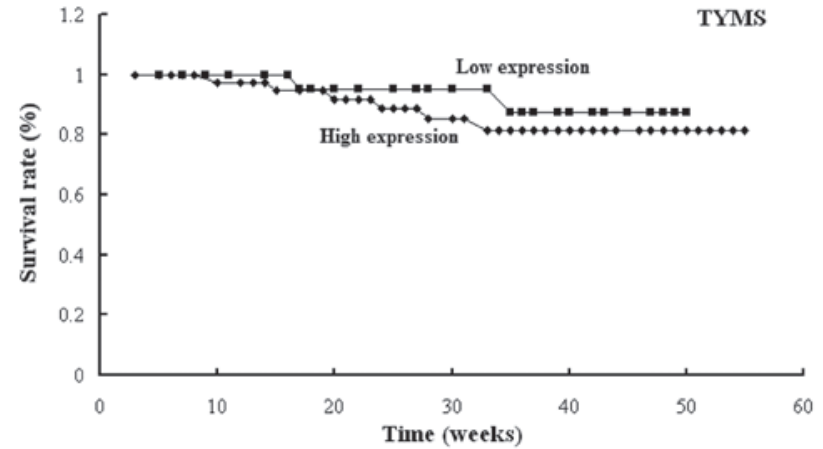

Figure 4. Survival curve following surgery in 72 patients with non-small cell lung cancer based on the mRNA expression of the TYMS gene. The one-year survival rates of the patients with high TYMS expression and low TYMS expression were 86.7 and $92.6 \%$, respectively. $\mathrm{P}=0.001$. TYMS, thymidylate synthase.

or TYMS expression and age, gender, smoking status, TNM stage or lymph node metastasis.

Correlation between ERCC1, RRM1, TUBB3 or TYMS mRNA expression and patient survival. Following surgery, all 72 patients with NSCLC were treated with platinum-based doublet chemotherapy. The median number of chemotherapy cycles was 4.5 . The follow-up duration was 379 days. There were eight cases of mortality. The overall survival rate was found to be $88.9 \%$. The results indicated that high expression of $E R C C l$ was associated with poor prognosis $(\mathrm{P}<0.001)$ and the one-year survival rates of the patients with high ERCCI expression and low ERCC1 expression were 78.6 and $95.4 \%$, respectively (Fig. 1). Similar results were found for the RRM1, $T U B B 3$ and TYMS genes (Figs. 2-4). High expression levels of $R R M 1, T U B B 3$ or TYMS were observed to be significantly associated with poor prognosis $(\mathrm{P}=0.001, \mathrm{P}=0.001$ and $\mathrm{P}=0.001$, respectively). The one-year survival rates of the patients were: 85 and $93.7 \%$ for those with high and low RRMI expression, respectively; 85.3 and $94.7 \%$ for those with high and low TUBB3 expression, respectively; and 86.7 and $92.6 \%$ in those with high and low TYMS expression, respectively.

\section{Discussion}

The combination of two cytotoxic drugs, for example platinum agents combined with non-platinum agents, including gemcitabine, has become the standard first-line treatment for patients with NSCLC (10). However, drug resistance is a major problem in NSCLC. Not all patients benefit from chemotherapy; thus, it is important to identify predictive markers for response to chemotherapy.

In the present study, the ERCC1, RRM1, TUBB3 and TYMS genes were investigated. ERCC1 is a key factor involved in nuclear excision repair for platinum-induced adducts. There is increasing evidence that reduced DNA repair capacity is correlated with enhanced survival with platinum-based chemotherapy $(11,12)$. However, certain studies have reported that the overexpression of ERCCl is associated with improved prognosis $(13,14)$. These contradictory reports may be due to regional disparity or differences in experimental procedures. The present study found that 
patients with NSCLC with high (positive) ERCCl expression had a poor prognosis and that the incidence rate of $E R C C 1$ expression in non-adenocarcinoma was higher than that in adenocarcinoma $(\mathrm{P}<0.05)$. Like ERCC1, RRM1 also has a role in DNA repair processes. RRM1 is the predominant cellular determinant of the efficacy of the nucleoside analog gemcitabine, and high expression of $R R M 1$ is associated with chemoresistance to gemcitabine-based therapy (15). Clinical studies have shown that $R R M 1$ expression is correlated with the expression of ERCC1 $(11,16)$. The present study indicated that RRMI expression may be correlated with $E R C C 1$ expression, but not TUBB3 and TYMS expression; however, no statistical significance was found $(r=0.193 ; \mathrm{P}>0.05$; data not shown). Statistical significance may increase with expansion of the sample size. These results indicate that the patients with NSCLC with high RRMI expression had a poor prognosis. TUBB3 is a structural protein and it has been reported that high expression of $T U B B 3$ is correlated with low response rates in patients with NSCLC treated with chemotherapy regimens using an anti-tubulin drug (17). However, studies have shown conflicting results, possibly due to variation in trial design or tumor stage analysis (18). The present study suggested that overexpression of $T U B B 3$ was associated with poor prognosis in patients with NSCLC. Furthermore, the incidence rate of $T U B B 3$ expression in high-median differentiation tissue samples was found to be significantly lower than that in the low differentiation tissue samples $(\mathrm{P}<0.05)$. TYMS is important for maintaining the dTMP pool for DNA synthesis and repair. Various studies have reported that high expression of TYMS is an unfavorable prognostic factor (19-21); however, it has also been reported to be a favorable prognostic factor (22). The findings of the present study indicate that patients with NSCLC with high TYMS expression had a poor prognosis and that the incidence rate of TYMS expression in high-median differentiation tissue samples was significantly lower than that in the low differentiation tissue samples $(\mathrm{P}<0.05)$.

In conclusion, although the sample size used in the present study was small, the findings indicate that ERCC1, RRM1, TUBB3 and TYMS are prognostic factors for survival and may be predictive biomarkers for platinum-based chemotherapy in patients with NSCLC. Detecting the mRNA expression of these four genes may be useful for predicting the efficacy of chemotherapy and may enhance survival in NSCLC.

\section{References}

1. Chen W, Zhang S and Zou X: Evaluation on the incidence, mortality and tendency of lung cancer in China. Thoracic Cancer 1: 35-40, 2010.

2. Jazieh AR, Bamefleh H, Demirkazik A, et al; MENA Lung Cancer Regional Guidelines Committee: Modification and implementation of NCCN guidelines on non-small cell lung cancer in the Middle East and North Africa region. J Natl Compr Canc Netw 8 (Suppl 3): S16-S21, 2010.
3. Li J, Li ZN, Yu LC, et al: Association of expression of MRP1, BCRP, LRP and ERCC1 with outcome of patients with locally advanced non-small cell lung cancer who received neoadjuvant chemotherapy. Lung Cancer 69: 116-122, 2010.

4. Rosell R, Danenberg K, Alberola V, et al; Spanish Lung Cancer Group: Ribonucleotide reductase messenger RNA expression and survival in gemcitabine/cisplatin-treated advanced non-small cell lung cancer patients. Clin Cancer Res 10: 1318-1325, 2004.

5. Peters GJ, Backus HH, Freemantle S, et al: Induction of thymidylate synthase as a 5-fluorouracil resistance mechanism. Biochim biophys Acta 1587: 194-205, 2002.

6. Azuma K, Sasada T, Kawahara A, et al: Expression of ERCC1 and class III beta-tubulin in non-small cell lung cancer patients treated with carboplatin and paclitaxel. Lung Cancer 64: 326-333, 2009.

7. Burkhart CA, Kavallaris M and Band Horwitz S: The role of beta-tubulin isotypes in resistance to antimitotic drugs. Biochim Biophys Acta 1471: O1-O9, 2001.

8. Flagella M, Bui S, Zheng Z, et al: A multiplex branched DNA assay for parallel quantitative gene expression profiling. Anal Biochem 352: 50-60, 2006.

9. Ren GJ, Zhao YY, Zhu YJ, et al: Tumor gene mutations and messenger RNA expression: correlation with clinical response to icotinib hydrochloride in non-small cell lung cancer. Chin Med J (Engl) 124: 19-25, 2011.

10. Crinò L, Weder W, van Meerbeeck $\mathrm{J}$ and Felip E; ESMO Guidelines Working Group: Early stage and locally advanced (non-metastatic) non-small-cell lung cancer: ESMO Clinical Practice Guidelines for diagnosis, treatment and follow-up. Ann Oncol 21 (Suppl 5): 103-115, 2010.

11. Zheng Z, Chen T, Li X, et al: DNA synthesis and repair genes RRM1 and ERCC1 in lung cancer. N Engl J Med 356: 800-808, 2007.

12. Lee KH, Min HS, Han SW, et al: ERCC1 expression by immunohistochemistry and EGFR mutations in resected non-small cell lung cancer. Lung Cancer 60: 401-407, 2008.

13. Gurubhagavatula S, Liu G, Park S, et al: XPD and XRCC1 genetic polymorphisms are prognostic factors in advanced non-small-cell lung cancer patients treated with platinum chemotherapy. J Clin Oncol 22: 2594-2601, 2004.

14. Zhou W, Gurubhagavatula S, Liu G, et al: Excision repair cross-complementation group 1 polymorphism predicts overall survival in advanced non-small cell lung cancer patients treated with platinum-based chemotherapy. Clin Cancer Res 10: 4939-4943, 2004.

15. Davidson JD, Ma L, Flagella M, et al: An increase in the expression of ribonucleotide reductase large subunit 1 is associated with gemcitabine resistance in non-small cell lung cancer cell lines. Cancer Res 64: 3761-3766, 2004

16. Ceppi P, Volante M, Novello S, et al: ERCC1 and RRM1 gene expressions but not EGFR are predictive of shorter survival in advanced non-small-cell lung cancer treated with cisplatin and gemcitabine. Ann Oncol 17: 1818-25, 2006.

17. Sève P, Reiman T, Lai R, et al: Class III beta-tubulin is a marker of paclitaxel resistance in carcinomas of unknown primary site. Cancer Chemother Pharmacol 60: 27-34, 2007.

18. Sève P and Dumontet C: Is class III beta-tubulin a predictive factor in patients receiving tubulin-binding agents? Lancet Oncol 9: 168-75, 2008.

19. Ceppi P, Rapa I, Lo Iacono M, et al. Expression and pharmacological inhibition of thymidylate synthase and Src kinase in nonsmall cell lung cancer. Int J Cancer 130: 1777-1786, 2012.

20. Grimminger PP, Schneider PM, Metzger R, et al: Low thymidylate synthase, thymidine phosphorylase, and dihydropyrimidine dehydrogenase mRNA expression correlate with prolonged survival in resected non-small-cell lung cancer. Clin Lung Cancer 11: 328-34, 2010.

21. Nakagawa T, Otake Y, Yanagihara K, et al: Expression of thymidylate synthase is correlated with proliferative activity in non-small cell lung cancer (NSCLC). Lung Cancer 43: 145-149, 2004.

22. Zheng Z, Li X, Schell MJ, et al: Thymidylate synthase in situ protein expression and survival in stage I nonsmall-cell lung cancer. Cancer 112: 2765-73, 2008. 